\title{
Understanding the Herschel-SPIRE bolometers
}

\author{
Adam L. Woodcraft ${ }^{a b}$, Hien Nguyen ${ }^{c}$, James Bock ${ }^{c}$, Matthew Griffin ${ }^{d}$, Bernhard Schulz ${ }^{e}$, Bruce \\ Sibthorpe $^{b}$, Bruce Swinyard $f^{f}$ \\ ${ }^{a}$ SUPA, Institute for Astronomy, Edinburgh University, Blackford Hill, Edinburgh EH9 3HJ, UK \\ ${ }^{b}$ UK Astronomy Technology Centre, Blackford Hill, Edinburgh EH9 3HJ, UK \\ ${ }^{c}$ Jet Propulsion Laboratory, 4800 Oak Grove Drive, Pasadena, CA 91109, USA \\ ${ }^{d}$ Cardiff School of Physics and Astronomy, Cardiff University, Queens Buildings, The Parade, \\ Cardiff CF24 3AA, UK \\ ${ }^{e}$ Infrared Processing and Analysis Center, California Institute of Technology, Pasadena, CA 91125, \\ USA \\ ${ }_{\text {Rutherford Appleton Laboratory, STFC, Chilton, Didcot, Oxon, OX11 OQX, UK }}$
}

\begin{abstract}
Bolometers are very simple devices. In principle, the behaviour of a bolometer can be described by a simple model along with a small number of parameters. The SPIRE instrument for the Herschel Space Observatory contains five arrays of NTD germanium spiderweb bolometers containing up to 139 pixels. We show from characterisation measurements on the ground using the flight read-out system that the bolometers follow the ideal model extremely well, are very stable, and that the read-out system is sufficiently well behaved to take advantage of this. Calibration should be greatly simplified by being able to take advantage of this behaviour.
\end{abstract}

Keywords: SPIRE, Herschel, sub-mm, observatory, satellite, detectors, bolometers, characterisation

\section{INTRODUCTION}

SPIRE (the Spectral and Photometric Imaging Receiver) is one of three instruments which will fly on the Herschel Space Observatory, ${ }^{1}$ an ESA mission with NASA participation. The instrument contains five bolometer arrays. Three form a three-band imaging photometer with bands centred at approximately 250, 350 and $500 \mu \mathrm{m}$ (referred to in this paper as the PSW, PMW and PLW arrays respectively), and the other two are part of an imaging Fourier Transform Spectrometer (FTS) covering the spectral range from 200 to $670 \mu \mathrm{m}$. The bolometer arrays, designed and constructed at the Jet Propulsion Laboratory (JPL) in the US, consist of feedhorn-coupled NTD (neutron transmutation doped) germanium spider-web bolometers cooled to $300 \mathrm{mK}$ by a closed cycle ${ }^{3} \mathrm{He}$ refrigerator.

The story of the bolometers has been told in a series of papers over the last decade. The initial concepts were described, ${ }^{2-4}$ followed by the results of tests on the "pathfinder" array used to demonstrate suitability for the SPIRE mission, ${ }^{5}$ and then measurements on the single array in the instrument qualification model (CQM). ${ }^{6}$ Measurements at JPL ${ }^{7,8}$ on each of the five arrays that subsequently formed the focal plane of the flight instrument have now been presented.

The latest chapter in the story is that the instrument build is now complete, and we are in the fortunate position of having been able to make extensive measurements on the arrays in the instrument itself, using the flight electronics, both with optical loading and with the detectors blanked from optical radiation. This has enabled us to characterise them to a very high level of accuracy. This paper describes the results from analysing load curve measurements (i.e. measurements of the bolometer voltage over a range of bias currents ${ }^{9,10}$ ) in the instrument. Other measurements such as those using the photometer calibration source (PCAL) ${ }^{11}$ also provide important information for characterisation of the arrays but are not discussed here. This paper also mentions only the photometer arrays, where analysis is more complete.

Further author information:

E-mail: adam.woodcraft@physics.org, Telephone: +44 8707651873

Copyright 2008 Society of Photo-Optical Instrumentation Engineers. This paper was published in Proc. SPIE 7020 and is made available as an electronic reprint with permission of SPIE. One print or electronic copy may be made for personal use only. Systematic or multiple reproduction, distribution to multiple locations via electronic or other means, duplication of any material in this paper for a fee or for commercial purposes, or modification of the content of the paper are prohibited. 


\section{MEASUREMENTS}

All five arrays were characterised individually at JPL. ${ }^{7,8}$ Apart from noise measurements, the measurements were carried out with a DC (direct current) bias. This is usual for characterisation measurements, since a DC bias system is straightforward to calibrate. The arrays were also extensively characterised in the SPIRE instrument itself at the Rutherford Appleton Laboratory. ${ }^{12}$ Measurements were taken in two configurations; one in which the arrays could view an internal blackbody (run "PFM4"), and one with the array blanked to all incident radiation (run "PFM5"), providing a truly dark environment. These measurements were made using the flight electronics, which use an AC (alternating current) bias in order to improve noise performance. The disadvantage is that various frequency-dependent effects appear which must be compensated for in order to obtain the true bolometer current and voltage. Other complications arise when carrying out characterisation measurements since the flight electronics are optimised for operation at a fixed bias current; for example, the readouts are designed to measure small voltage differences from a fixed offset value, enabling effectively 20 bit sampling from a 16-bit ADC (analogue to digital converter).

All these effects must be correctly allowed for in order to carry out characterisation measurements in the instrument. The obvious advantage is that the measurements are directly applicable to measurements in flight. For example, the gains of the JFETs vary slightly between channels; this has to be explicitly and accurately allowed for when attempting to apply characterisation measurements taken outside the instrument, and is believed to be the source of discrepancies reported previously ${ }^{6}$ between load curves taken in the instrument and at JPL.

In addition, instrument level measurements give information on the behaviour and stability of the detectors in the environment of the instrument as opposed to a test cryostat, and also on the performance of the flight read-out systems themselves. Finally, far more measurements were made with the arrays in the instrument than beforehand, giving a much larger body of data to use to examine stability and consistency of measurements.

\section{CONSISTENCY}

The behaviour of a bolometer is normally described with a model known as the ideal bolometer model, or thermal model. ${ }^{9} 10$ This considers the bolometer as a simple system consisting of a thermistor mounted on an absorber of optical power, linked to a perfect heat sink by a weak thermal link. The model essentially makes two assumptions. One is that the bolometer thermistor resistance $R$ is a simple function of temperature, $T$ :

$$
R=R^{*} \exp \sqrt{\frac{T_{g}}{T}}
$$

where $R^{*}$ and $T_{g}$ are constants. (In fact for NTD germanium, this equation does not necessarily follow a dependence on $\sqrt{(1 / T)}$; however, it does provide good fits for the SPIRE bolometers ${ }^{13}$ ).

The constants in Eq. (1) for each bolometer were determined by taking load curves at a variety of heat sink temperatures with the bolometers blanked from optical radiation (run PFM5). The resistance at the heat sink temperature was then determined by the resistance from the load curve in the limit of zero bias. ${ }^{14}$ Once this has been determined, it is possible to examine the consistency of bolometer measurements without invoking the thermal model. This can be done by using numerical differentiation to plot a load curve in the form $G(T)=d P / d T$ as a function of bolometer (absorber) temperature, $T$, where $G$ is the thermal conductance and $P$ is the electrical power (given by $V I$ where $V$ and $I$ are bolometer voltage and current respectively). Since $G(T)$ is a physical property of the bolometer, it does not depend on the heat sink temperature, nor on the optical loading. It is therefore possible to directly compare a set of load curves taken under different conditions on the same bolometer; this is not possible if they are plotted in the usual form of voltage against current.

This is shown for one bolometer from each array in Fig. 1. These measurements were taken over two cooldowns (PFM4 and PFM5) and despite the fact that they were taken over a period of four months, during which environmental conditions varied, the results show a high level of consistency. Measurements were also taken at different bias frequencies, showing that frequency-dependent effects have been removed well. The bolometers and readout systems are clearly extremely stable.

These results also show that resistance is a function of temperature only, and not on the bolometer voltage as is seen with similar detectors operating at lower temperatures. ${ }^{15}$ (The deviations at the lowest temperature in each load curve come from attempting to find the derivative $d P / d T$ where the difference in both $P$ and $T$ between adjacent data points is extremely small, and errors therefore blow up.) 

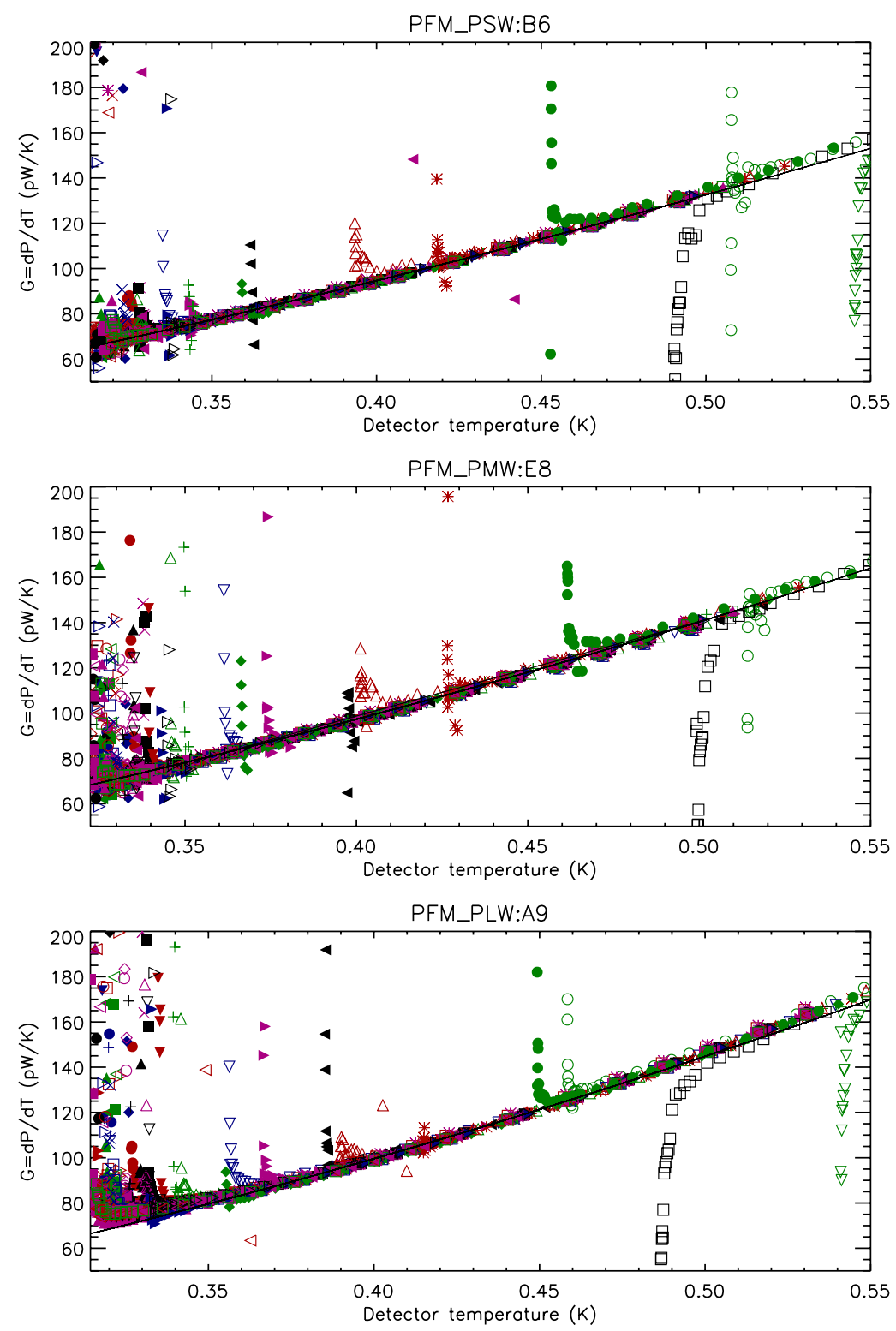

Figure 1. Bolometer thermal conductance $G$ as a function of absorber temperature for many load curve measurements for a single bolometer in each array. The reason for choosing these particular bolometers is given in section 4 . The solid black line shows the fit from thermal model fits to a single load curve (Section 4).

\section{MODELLING}

Further analysis can be carried out using the thermal model. This is often used to characterise bolometers during the development process, in order to produce a bolometer with the appropriate characteristics. However, the usefulness does not end once the bolometers have been constructed. Being able to accurately model the bolometers in flight has many advantages, such as being able to predict their behaviour under different conditions with a simple simulator program, and to aid calibration of the detectors.

In addition to Eq. (1), the thermal model makes the assumption that the conductance $G$ of the weak thermal link at 

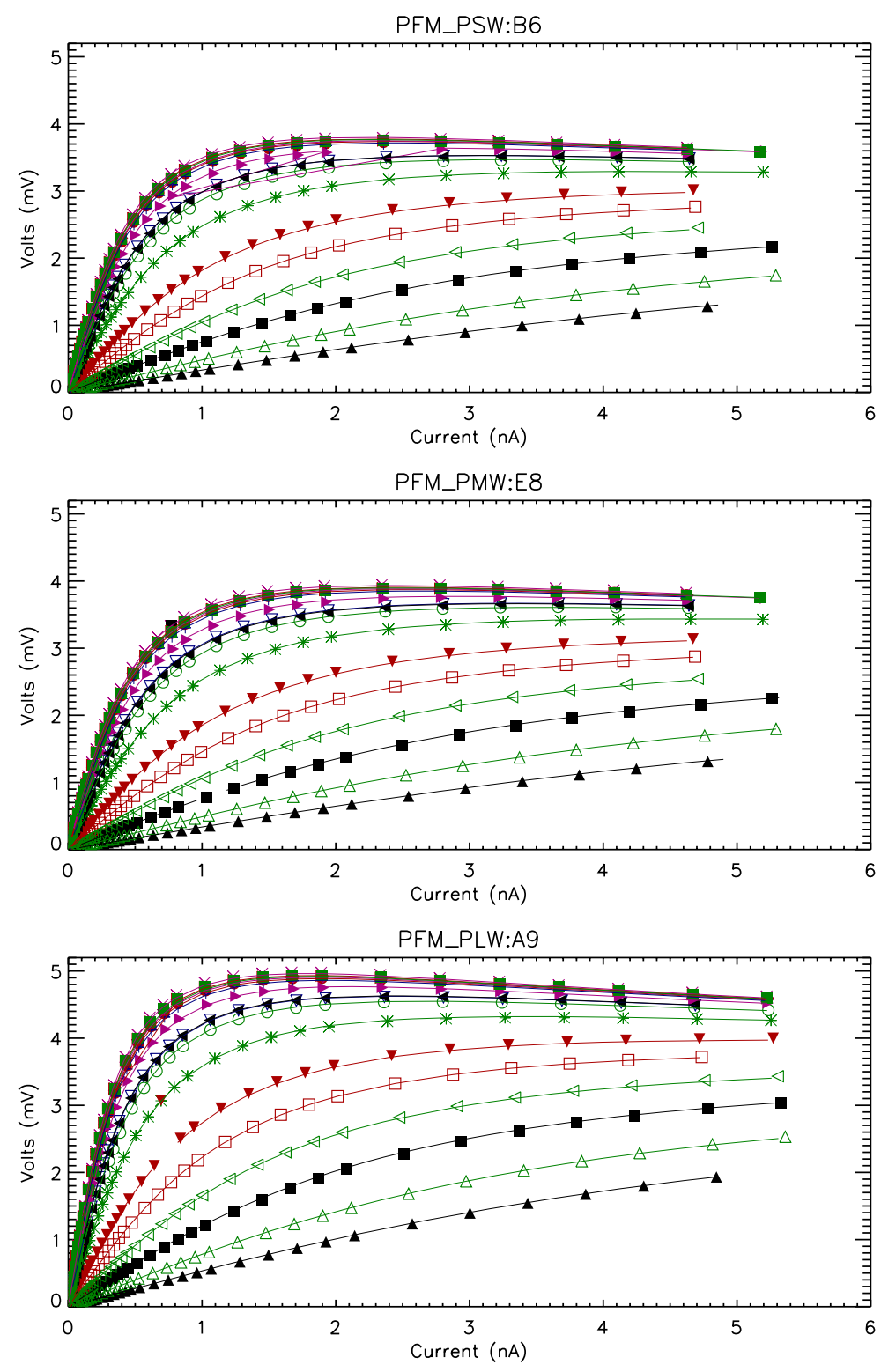

Figure 2. A set of load curves for a variety of heat sink temperatures with the bolometers optically blanked (run PFM5). The solid lines show the prediction from the thermal model, using parameters obtained from a single load curve (observation ID 23FD).

absorber temperature $T$ follows a power-law, and thus can be represented as

$$
G(T)=G_{0} T^{\beta}
$$

where $G_{0}$ and $\beta$ are constants. (By expressing the thermal model in terms of voltage as a function of current, it is possible to make the model look exceedingly complex. However, fundamentally it is this simple.) Having determined the constants in Eq. (1) from a set of load curves, the constants in Eq. (2) can be determined by non-linear fitting to electrical power as a function of bolometer temperature for any load curve.

Figure 2 shows the results from thermal model fits to load curves on the same bolometers shown in Fig. 1. These mea- 

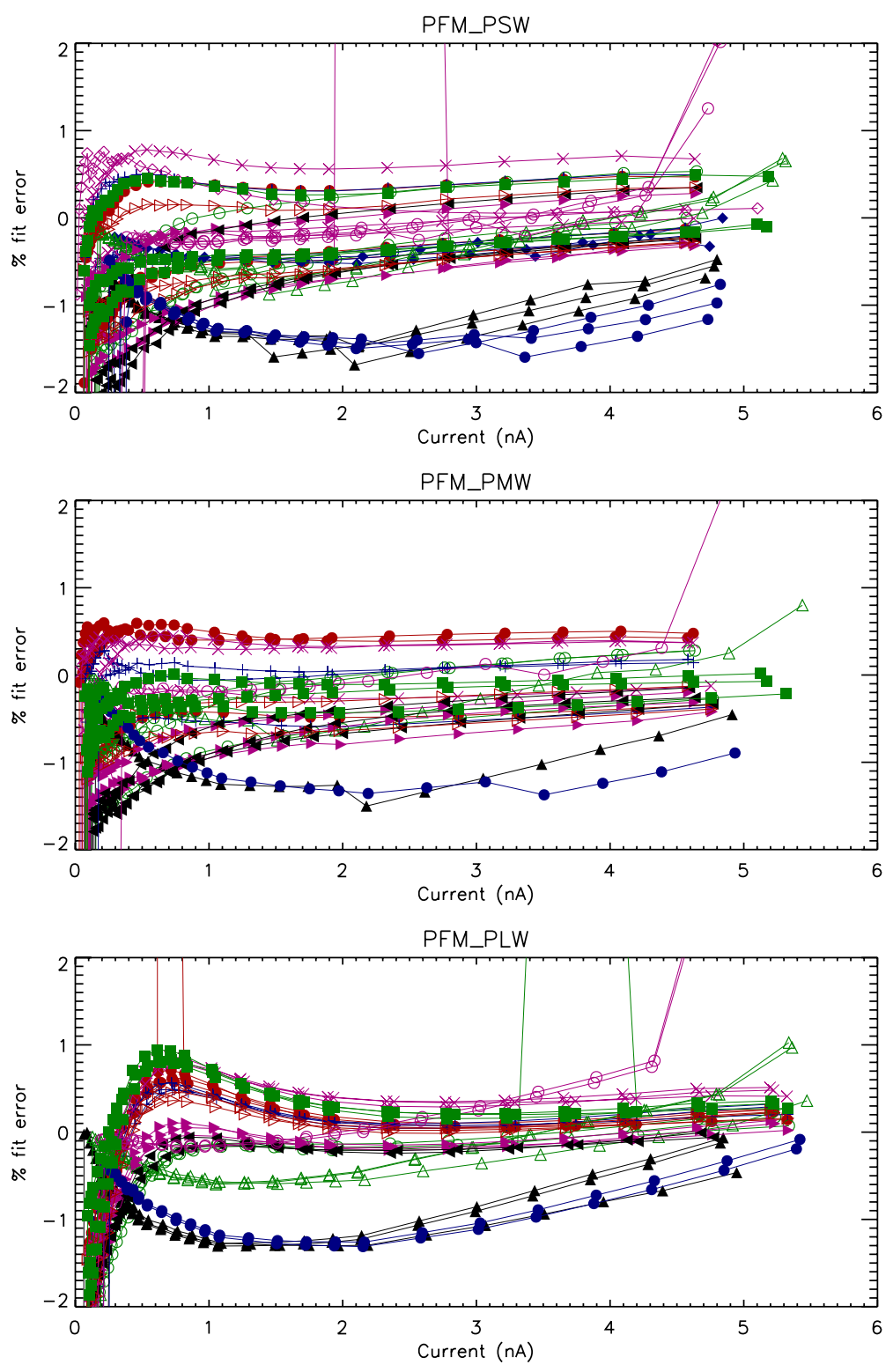

Figure 3. Error in thermal model fits to the sets of load curves shown in Fig. 2, for three bolometers in each array (PSW:B6,D15 \& F4, PMW:D9,E8 \& F4, PLW:A9,C7 \& E2). Measurements made (simultaneously) under the same conditions on different bolometers are shown with the same symbols.

surements were obtained with the bolometers optically blanked (run PFM5). It is common when characterising bolometers to carry out the thermal model fits separately to each load curve for a given bolometer. However, the model parameters should not vary as a function of the heat sink temperature (or anything else for that matter). It should therefore be possible to obtain the parameters from a single load curve and then apply them to a family of load curves taken at different heat sink temperatures. This was done in Fig. 2; for each bolometer the parameters were obtained from a load curve at a heat sink temperature of approximately $300 \mathrm{mK}$. It is clear that this procedure produces good fits to the load curves, and that the thermal model is therefore a very good description of the behaviour of the bolometers. In fact, the results in Fig. 1 tell 

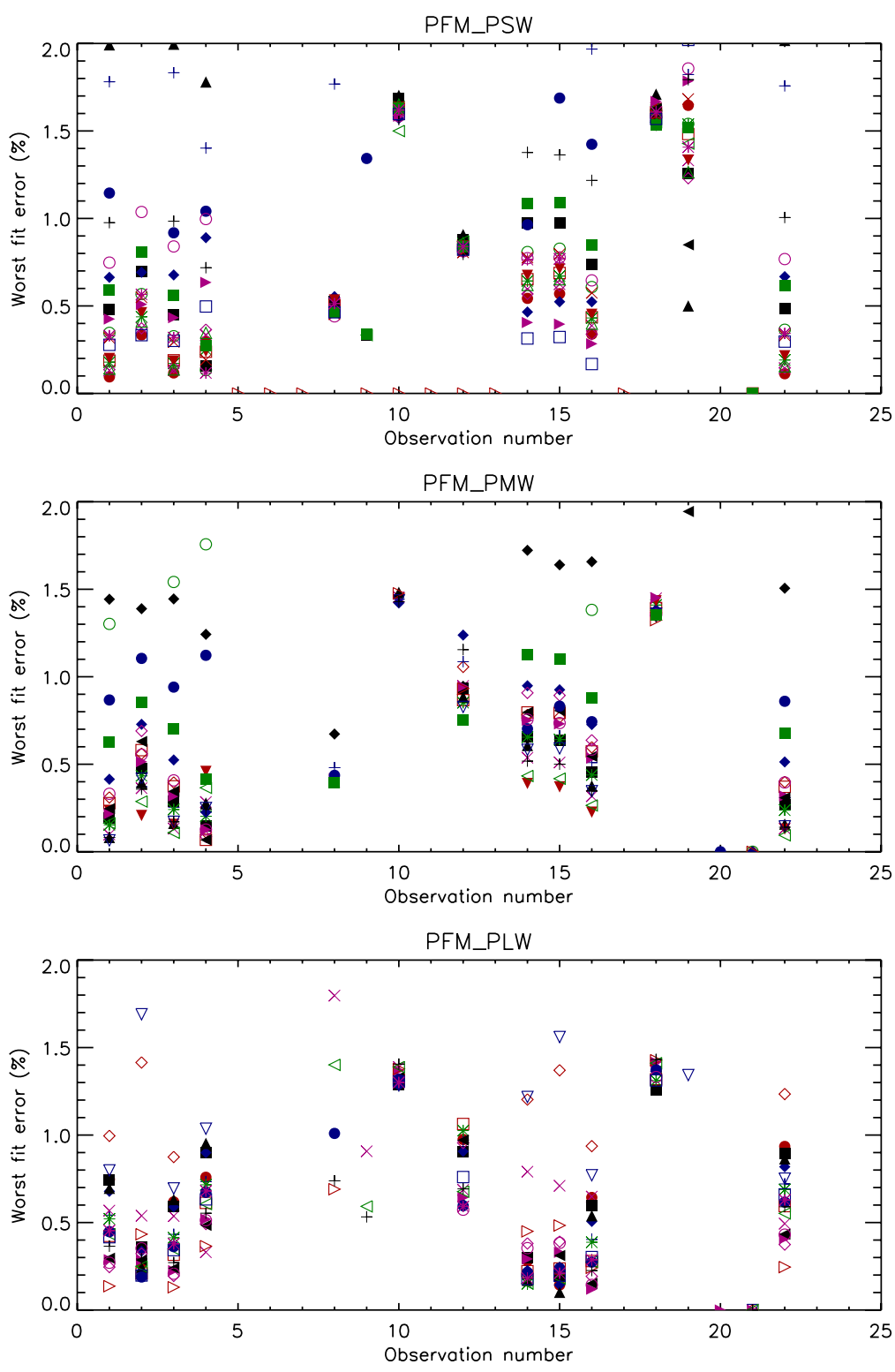

Figure 4. Maximum error between measurements and the thermal model fit for 25 bolometers from each array, for all the load curves in the optically blanked run (PFM5).

us that this should be the case, since they show that $G(T)$ follows a power-law as assumed in Eq. (2).

Of course, the SPIRE arrays have many more pixels than the three shown here. Analysing the results from all the pixels presents something of a challenge, as does showing the results in a meaningful manner. The next three figures represent an attempt to do so. Figure 3 shows the percentage error in the thermal model fits to three bolometers for each array, for blanked load curves at different stage temperatures as shown in Fig. 2. The errors are generally within $\pm 1 \%$.

Figure 4 shows the maximum errors in the fit for 25 bolometers chosen randomly from each array (ignoring bias currents below $1 \mathrm{nA}$, where the voltage becomes small and the percentage errors relatively large; this is well below any bias value that would be used in flight). Figure 5 shows the errors for a single measurement (observation number 1 in 

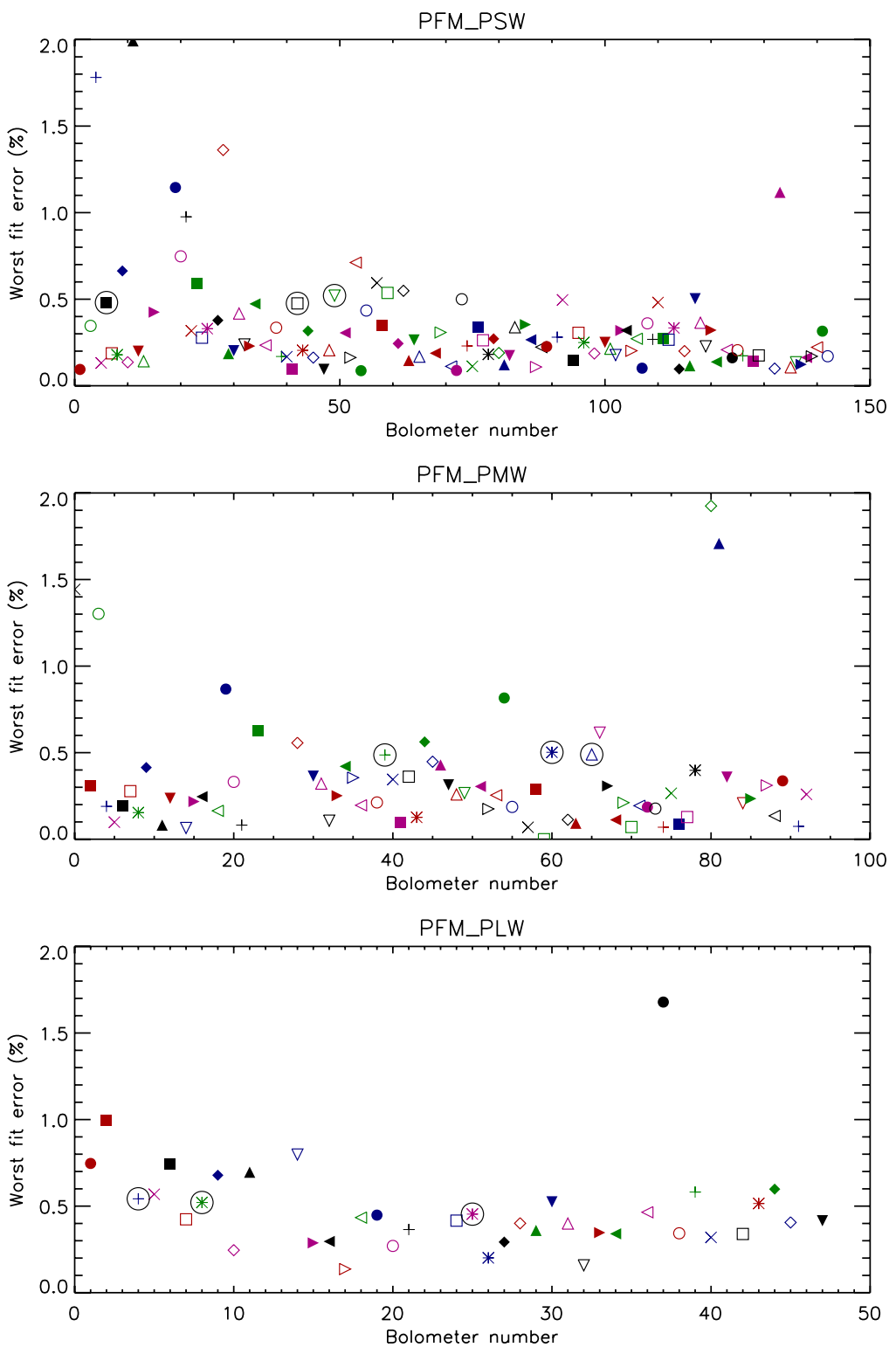

Figure 5. Maximum error between measurements and the thermal model fit for all bolometers, for a single load curve in the optically blanked run (Observation ID 20F7).

Fig. 4), as a function of bolometer number, for all the bolometers.

The errors cluster around a small range, with a few outliers with larger errors (these outliers are probably caused by the odd bad data point, and it should be possible to improve the accuracy by further analysis). We have chosen a set of three bolometers from each array with errors around $0.5 \%$. In other words they show the highest errors, apart from the outliers, and could be considered "typical results" in a rather pessimistic sense; these are ringed in black in Fig. 5. These bolometers (or a single one from each array chosen at random) were used in Figs. 1, 2, 3, 6 and 7.

Now, so far we have shown that the thermal model fits are very accurate if expressed in terms of voltage. However, it does not follow that the errors will be equally small if the load curves are plotted in a different manner. For example, 

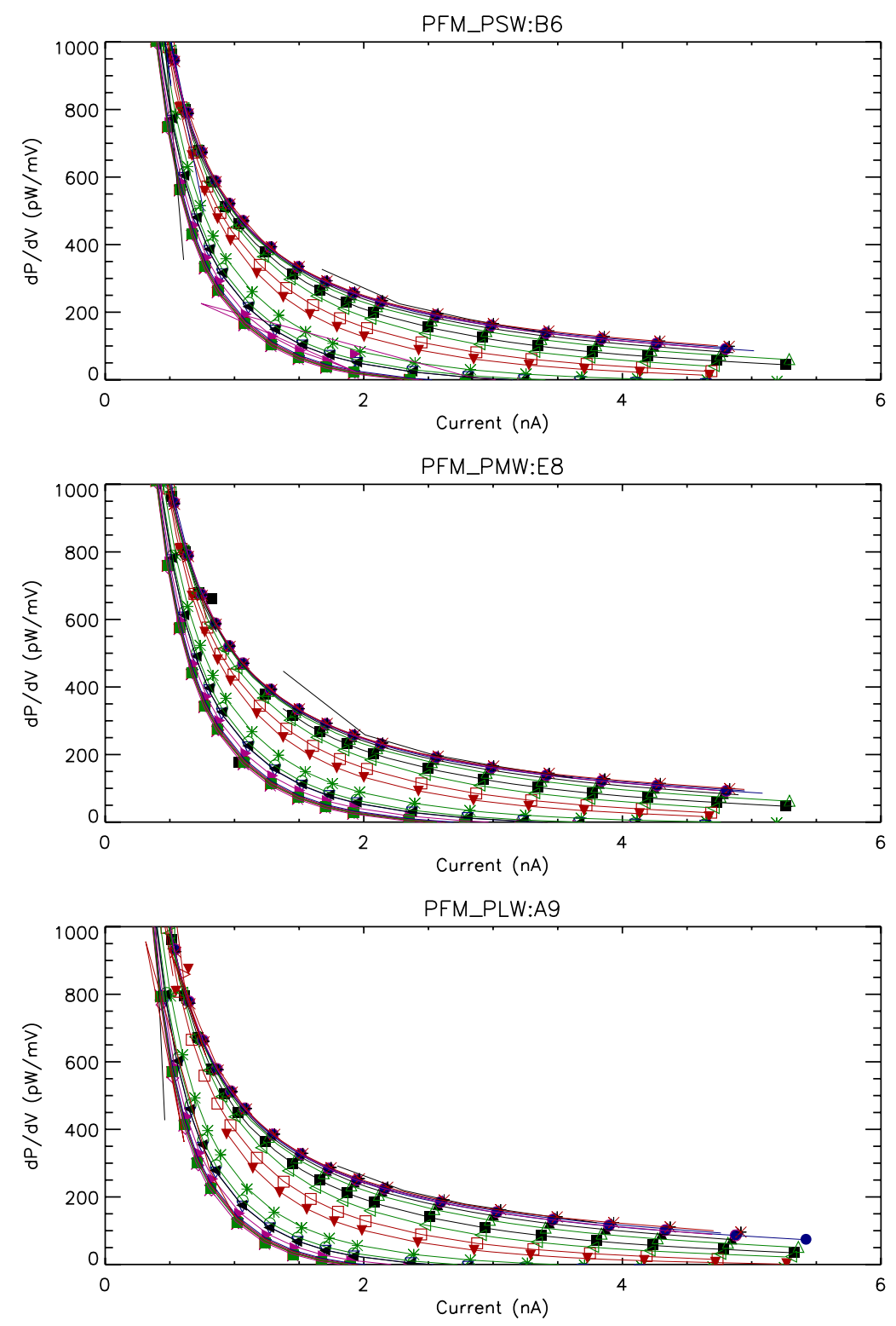

Figure 6. Comparison between $d V / d P$, where $V$ is bolometer voltage and $P$ is electrical power, obtained by numerical differentiation of measured data (points) and thermal model predictions (lines), for one bolometer from each array.

a thermal model fit can appear to be in very good agreement with the data if plotted in terms of voltage as a function of current, but show poor agreement when plotted as $d P / d T$ as in Fig. $1 .{ }^{10}$ In this case, we see good agreement when plotted in this form, as can be seen from Fig. 1 (the thermal model fit line is hard to see because it agrees with the data points so well).

However, for astronomical observations we are interested in how the voltage varies with absorbed power, not with bias current or thermal conductance. Figure 6 shows $d P / d V$, obtained by numerical differentiation, where $V$ is bolometer voltage and $P$ is electrical power (voltage $\times$ current), for both the measured data and the thermal model fits. Again, the agreement is encouragingly good. 

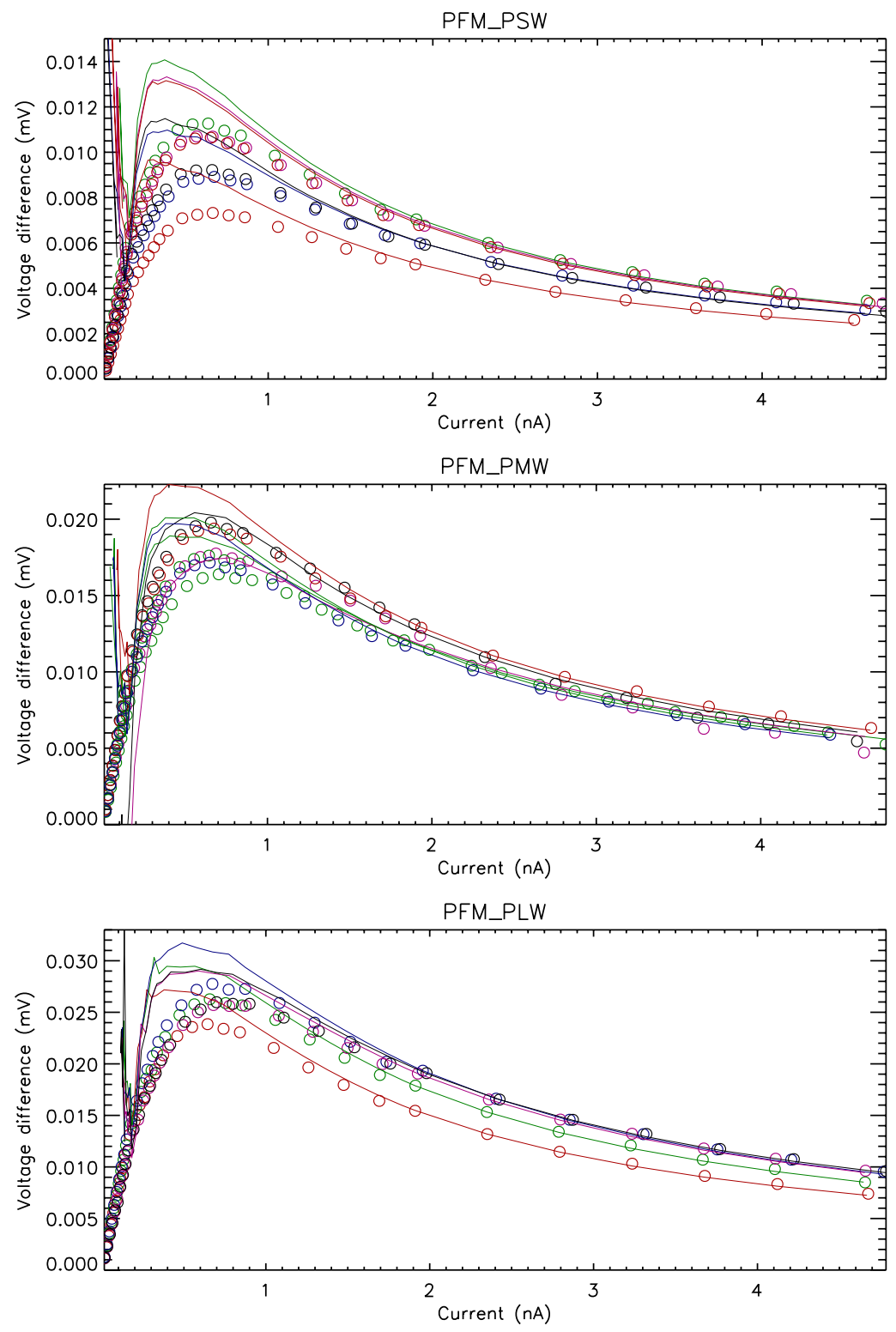

Figure 7. Comparison between the difference in detector voltage at a given bias current for load curves obtained under slightly different optical powers, obtained from measured data (points) and thermal model predictions (lines), for one bolometer from each array. (The observation IDs are 17D1 and 17D5.)

The effect of varying electrical power is not necessarily the same as varying optical power. To demonstrate the effect of varying optical power, Fig. 7 shows the difference in voltage for a pair of load curves taken with the bolometers observing the internal blackbody at two slightly different temperatures, and therefore corresponding to two slightly different optical powers. Again, the measured values are compared to the thermal model fits. Once more, the agreement is good for currents above $1 \mathrm{nA}$, where the bolometers will be biased in operation. It is worth noting here that the model parameters used were taken not only from a different load curve, but one measured several months later and after warming up the instrument and cooling it down again. 
Overall, then, the thermal model gives an extremely accurate and useful description of the behaviour of the bolometers.

\section{CONCLUSIONS}

The bolometers and their accompanying readout systems are extremely stable over long time periods, even when operated on the ground with varying environmental conditions.

We have overcome the complexities of the AC biased readout system in order to be able to characterise the arrays while in the instrument. Combining the results of two cooldowns, one in which the arrays could view an internal blackbody and another in which they were blanked from optical radiation has enabled a thorough characterisation using the flight electronics. This has shown that the bolometers can be modelled to high accuracy using the ideal bolometer model. This gives us the ability to accurately predict their performance under different conditions encountered in flight, enabling us to allow for effects such as changes in heat sink temperature and for non-linearity in a non-empirical manner, and perhaps ultimately to use the bolometer model to calibrate the detectors.

\section{REFERENCES}

[1] Pilbratt, G. L., "Herschel mission: status and observing opportunities," Proc. SPIE 5487, 401-412 (2004)

[2] Bock, J. J., DelCastillo, H. M., Turner, A. D., Beeman, J. W., Lange, A. E., and Mauskopf, P. D., "Infrared bolometers with silicon nitride micromesh absorbers," Proc. 30th ESLAB Symp. "Submillimetre and Far-Infrared Space Instrumentation”, 24-26 September 1996, ESA SP-388, 119-122 (1996).

[3] Mauskopf, P. D., Bock, J. J., Castillo, H. D., Holzapfel, W. L., and Lange, A. E., "Composite infrared bolometers with $\mathrm{Si}_{3} \mathrm{~N}_{4}$ micromesh absorbers," Appl. Opt. 36(4), 765-771 (1997).

[4] Bock, J. J., Glenn, J., Grannan, S. M., Irwin, K. D., Lange, A. E., LeDuc, H. G., and Turner, A., "Silicon nitride micromesh bolometers for SPIRE," Proc. SPIE 3357, 297-304 (1998).

[5] Turner, A. D., Bock, J. J., Beeman, J. W., Glenn, J., Hargrave, P. C., Hristov, V. V., Nguyen, H. T., Rahman, F., Sethuraman, S., and Woodcraft, A. L., "Silicon nitride micromesh bolometer array for submillimeter astrophysics," Appl. Opt. 40, 4921-4932 (2001).

[6] Lim, T., Swinyard, B., Aramburu, A., Bock, J., Ferlet, M., Griffin, D., Griffin, M., Hargrave, P., King, K., Leeks, S., Naylor, D., Ronayette, S., Sawyer, E., Schulz, B., Sidher, S., Spencer, L., Smith, D., and Woodcraft, A., "First Results From Herschel-SPIRE Performance Tests," Proc. SPIE 5487, 460-468 (2004).

[7] Nguyen, H. T., Bock, J. J., Ringold, P., Battle, J., Elliott, S. C., Turner, A. D., Weilert, M., Hristov, V. V., Schulz, B., Ganga, K., Zhang, L., Beeman, J. W., Ade, P. A. R., and Hargrave, P. C., "A report on the laboratory performance of the spectroscopic detector arrays for SPIRE/HSO,” Proc. SPIE 5498, 196-207 (2004).

[8] Nguyen, H. T., Ringold, P., Ade, P. A. R., Battle, J., Beeman, J. W., Bock, J. J., Elliott, S. C., Hargrave, P. C., Schulz, B., Turner, A. D., Hristov, V. V., Weilert, M., and Zhang, L., "A report on laboratory performance of the bolometric detector arrays for SPIRE/HSO: Part II,” Proc. SPIE 6275 (2006).

[9] Sudiwala, R. V., Griffin, M. J., and Woodcraft, A. L., "Thermal modelling and characterisation of semiconductor bolometers," Int. J. Inf. Mill. Waves 23(4), 545-573 (2002).

[10] Woodcraft, A. L., "An introduction to semiconductor bolometer modelling," Tech. Rep., Cardiff University (2005). http://reference.lowtemp.org/woodcraft/bologuide.pdf.

[11] Hargrave, P., Waskett, T., Lim, T., and Swinyard, B., "Performance of flight-model on-board calibration sources on Herschel-SPIRE," Proc. SPIE 6275, 627514-1-627514-12 (2006).

[12] Lim, T., Swinyard, B., Griffin, M., Aramburu, A., Baluteau, J.-P., Bock, J., Ferlet, M., Fulton, T., Griffin, D., Guest, S., Hargrave, P., King, K., Leeks, S., Naylor, D., Polehampton, E., Rizzo, D., Sawyer, E., Schulz, B., Sidher, S., Spencer, L., Smith, D., Nguyen, H. T., Valtchanov, I., Waskett, T., and Woodcraft, A., "Preliminary results from Herschel-SPIRE flight instrument testing," Proc. SPIE 6265 62650F-1-62659F-10 (2006).

[13] Woodcraft, A. L., Sudiwala, R. V., Wakui, E., and Piat, M., "Using experimental data to constrain theories of hopping conduction in NTD germanium," Physica B 329-333, 1300-1301 (2003).

[14] Woodcraft, A. L., Sudiwala, R. V., Griffin, M. J., Wakui, E., Maffei, B., Tucker, C. E., Haynes, C. V., Gannaway, F., Ade, P. A. R., Bock, J. J., Turner, A. D., Sethuraman, S., and Beeman, J. W., "High precision characterisation of semiconductor bolometers," Int. J. Inf. Mill. Waves 23(4), 575-595 (2002). 
[15] Piat, M., Torre, J.-P., Bréelle, E., Coulais, A., Woodcraft, A., Holmes, W., and Sudiwala, R., "Modeling of Planckhigh frequency instrument bolometers using non-linear effects in the thermometers," Nucl. Instrum. Methods in Phys. Res. A $559558-590$ (2006). 